\title{
Benign metastasizing leiomyoma presenting as multiple cystic pulmonary nodules: a case report
}

Yeong Hun Choe 1,3 , So Yeon Jeon ${ }^{1}$, Yoon Chae Lee ${ }^{1}$, Myung Ja Chung ${ }^{2,3}$, Seung Yong Park ${ }^{1,3}$, Yong Chul Lee ${ }^{1,3^{*}}$ and So Ri Kim ${ }^{1,3^{*}}$ (D)

\begin{abstract}
Background: Benign metastatic leiomyoma (BML) is an extremely rare disease. Although uterine leiomyomas are benign histologically, they can metastasize to distant sites. While the incidence is very low, the lung is the organ most frequently affected by BML. Pulmonary BML usually presents as numerous well-defined nodules of various sizes, while the cavitary or cystic features in the nodules are rarely observed on radiologic images.

Case presentation: A 52-year-old woman complained of cough and dyspnea for one month. She had been previously diagnosed with uterine leiomyoma and had undergone total hysterectomy about 14 years prior. Highresolution computed tomography $(C T)$ images showed that there were multiple cystic nodules of various sizes in both lungs. Pathologic examination revealed that the pulmonary nodule had complex branching glandular structures lined by a single layer of simple cuboidal to columnar epithelium that was surrounded by abundant spindle cells. Additional immunohistochemistry data suggested that pulmonary nodule diagnosis was BMLassociated uterine leiomyoma.

Conclusion: In this report, we introduce an interesting case of pulmonary BML that presented as a combination of various kinds of nodules including simple round nodules, simple cysts, and cysts with a solid portion, which are very rare radiologic features of BML in lung. In addition, when the patient is a woman of reproductive age, physicians should meticulously review the gynecological history and suspect BML when there are various cystic pulmonary lesions.
\end{abstract}

Keywords: Benign metastatic leiomyoma, Lung, Multiple cysts

\section{Background}

Benign metastasizing leiomyoma (BML) is a very rare condition that has been reported in association with uterine leiomyoma [1]. BML usually affects women of reproductive age with a history of uterine myoma. Although uterine leiomyomas are histologically benign, they can metastasize to distant sites such as lung, skin, bone, mediastinum, lymph node, muscular tissue, heart, and retroperitoneum $[2,3]$. Among them, lung is the organ most affected.

Patients with pulmonary BML are usually asymptomatic. Therefore, the lesions are frequently detected on radiologic

\footnotetext{
* Correspondence: leeyc@jbnu.ac.kr; sori@jbnu.ac.kr

'Department of Internal Medicine, Chonbuk National University Medical School, San 2-20, Geumam-dong, Deokjin-gu, Jeonju, Jeonbuk 561-180, South Korea

Full list of author information is available at the end of the article
}

examination during medical check-up. Common radiographic features of pulmonary BML are multiple pulmonary solid nodules of various sizes [4-9]. However, there are few reports of BML presenting as cystic or cavitary features.

Herein, we describe a case of pulmonary BML exhibiting an intriguing radiologic finding on chest computed tomography $(\mathrm{CT})$, a combination of various nodules having a cystic nature as well as mixed solid and cystic features.

\section{Case presentation}

A 52-year-old woman complained of cough and dyspnea for one month. There was no history of weight loss, hemoptysis, chest pain, smoking, or environmental and/or drug exposure. Physical and laboratory examination 
including spirometric analysis did not show any relevant abnormality. About 14 years prior, the patient was diagnosed with uterine leiomyoma and had undergone total hysterectomy.

Chest X-ray showed no definite lesions in either of the lung fields. However, high resolution CT showed multiple cystic nodules of variable size $(1.0 \mathrm{~cm}$ to $1.4 \mathrm{~cm}$ in diameter) in both lungs (Fig. 1). Some nodules were mixed with solid portions, but pure solid nodules were also observed. After reviewing the chest imaging, we performed several diagnostic tests to define possible etiologies for these multiple cystic lesions, including infection, malignancy, and immunologic diseases. There was no definitive endobronchial lesion on bronchoscopic examination, and bronchial washing cytology was negative for malignancy. Bronchoalveolar lavage cultures were negative for bacteria, mycobacterium, virus, and fungi. For pathologic diagnosis, we performed wedge resection of the pulmonary nodule in the right middle lobe. The surgical specimen contained a well-demarcated cystic nodule possessing a pale yellow-colored solid portion. Grossly, the nature of the nodule was firm with a size of $1.2 \mathrm{~cm} \times 1.0 \mathrm{~cm}$ in diameter. Microscopic findings showed complex branching glandular structures lined by a single layer of simple cuboidal to columnar epithelium were surrounded by abundant spindle cells. There was no atypia or mitotic activity in either the epithelial or the spindle cell components (Fig. 2). Immunohistochemically, the epithelial lining cells were positively stained for epithelial membrane antigen (EMA) and thyroid transcription factor (TTF)-1. In addition, the tumor cells contained smooth muscle actin (SMA). Desmin, estrogen receptor (ER), and progesterone receptor (PR) were also found in the cells of the nodule, while tumor cells were negative for HMB- 45 and S100 (Fig. 3). Based on these pathologic features, we diagnosed the pulmonary nodules as BML associated with uterine leiomyoma. We decided to monitor the patient's pulmonary lesions without further treatment. She had no aggravation of BML for 6 years since diagnosis.

\section{Discussion}

Pulmonary BML was first identified by Steiner in 1939 [1], and as he believed it to be a primary lung neoplasm, BML was first referred to as fibroleiomyomatous hamartoma. BML is a rare pathologic condition and difficult to diagnose mainly due to the lack of clinical suspicion. BML occurs in young premenopausal women aged 35 to 55 years with a history of uterine myoma or surgery such as hysterectomy or myomectomy. Although about 130 BML cases have been reported previously [2, 10-14], cystic pulmonary BML has not been reported. Based on information from previous cases, the mean interval between hysterectomy and the development of BML is 14.9 years. Most of the patients have favorable outcomes with a calculated median survival of 94 months [3].

Typical radiologic findings of pulmonary BML are numerous well-defined solid pulmonary nodules sized from a few millimeters to several centimeters, whereas there are few reports of cystic or cavitary nodular manifestation [4-9]. We previously reported that the typical radiologic feature was a round shaped solid pulmonary nodule on chest CT [10]. To our knowledge, simultaneous presentation of various radiologic features of pulmonary BML nodules like this current case has not been reported.

In clinical practice, physicians should carefully consider the following etiologies for multiple cystic lesions: neoplastic origin, infections by bacteria, fungi, and/or parasites, immunologic diseases, pulmonary embolism,

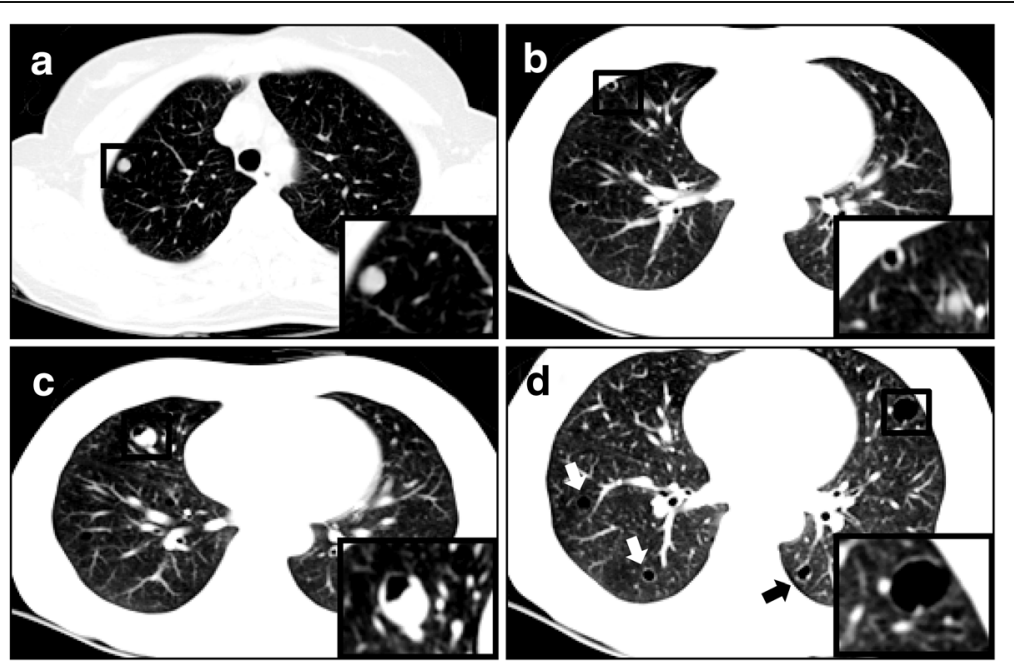

Fig. 1 Chest CT reveals multiple cystic nodules. a A 6 mm-sized round nodule in the right upper apical segment. b A 1 cm-sized cystic lesion with a thin wall in both lungs' parenchyma. c A 1 cm-sized cystic and well demarcated lesion with solid portion in the right middle lobe. $\mathbf{d}$ Multiple cystic nodules (arrows) with various sizes are in both lungs. CT: Computed tomography 

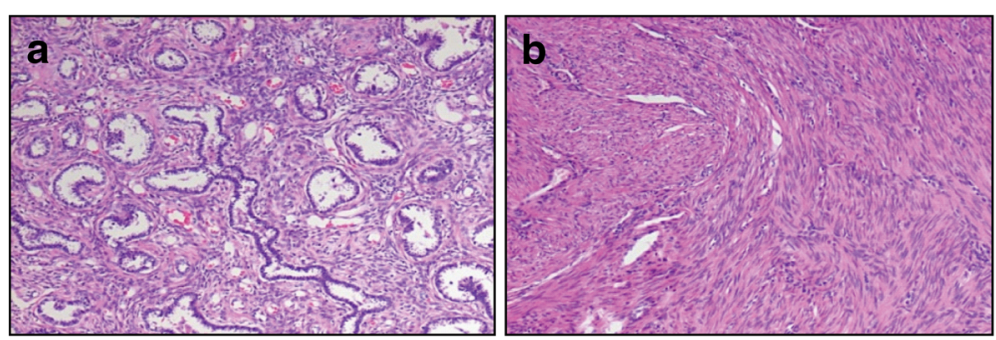

Fig. 2 Representative H\&E-stained sections of the pulmonary nodule (a) and the uterine mass (b). a The pulmonary nodule showed complex branching glandular structures lined by a single layer of simple cuboidal to columnar epithelium surrounded by abundant spindle cells. There was no atypia or mitotic activity in either the epithelial or the spindle cell components (H\&E staining, $\times 100$ ). $\mathbf{b}$ The uterine mass resected 14 years prior consisted of myometrium-derived smooth muscle cells and extracellular matrix

pneumoconiosis, localized bronchiectasis, pulmonary lymphangioleiomyomatosis (LAM), pulmonary Langerhans cell histiocytosis (PLCH), and so on, since there are many more common causes than pulmonary BML. Moreover, solid contents within a cavity may be a clue that the cystic lesions were caused by infectious processes such as invasive aspergillosis or by necrotic cancer [15]. In this present case, we decided to confirm the etiology of nodular lesions pathologically, considering these differential diagnoses. The site of the target nodule on the right middle lobe was suitable for wedge resection to obtain adequate tissues for diagnosis.

Pathologically, BML exhibits extrauterine proliferations of bland smooth muscle cells and mitotic inactivity $[16,17]$. There is no evidence of nuclear pleomorphism or necrosis in BML. BML usually shows a similar histologic appearance to that of a primary benign uterine tumor, i.e., leiomyoma. Therefore, immunohistochemically positive staining for ER and/or PR provides the diagnostic keys for BML. Exclusion of LAM is important in the diagnosis of cystic BML because it is a representative lung disorder that presents with multiple pulmonary cavities in middle-aged women. Both BML and LAM show some similarities; smooth muscle cell proliferation and expression of ER and PR in tumor cells [18]. However, pathologic patterns and immunochemical properties are helpful to distinguish BML from LAM. Smooth muscle cells proliferate with a peribronchial pattern in BML, while LAM shows proliferation of atypical smooth muscle cells along with lymphatics, blood vessels, and small airways. Moreover, the positivity of HMB-45 in the tumor cells is more important than anything else [19]. In the current case, the tumor was composed of abundant spindle cells that showed positivity for SMA, desmin, ER, and PR and negativity for HMB-45 and S100, suggesting a pathologic diagnosis of BML.

Some studies have shown cytogenetic abnormalities in BML [20]. If BML is hypothesized to arise from uterine myoma, demonstration of shared genetic abnormalities between the primary and metastatic lesions may provide valuable information for a diagnosis of BML, especially when morphological and histopathologic features are unusual. Examples of proposed abnormalities include $19 \mathrm{q}$ and $22 \mathrm{q}$ terminal deletion, X-chromosome

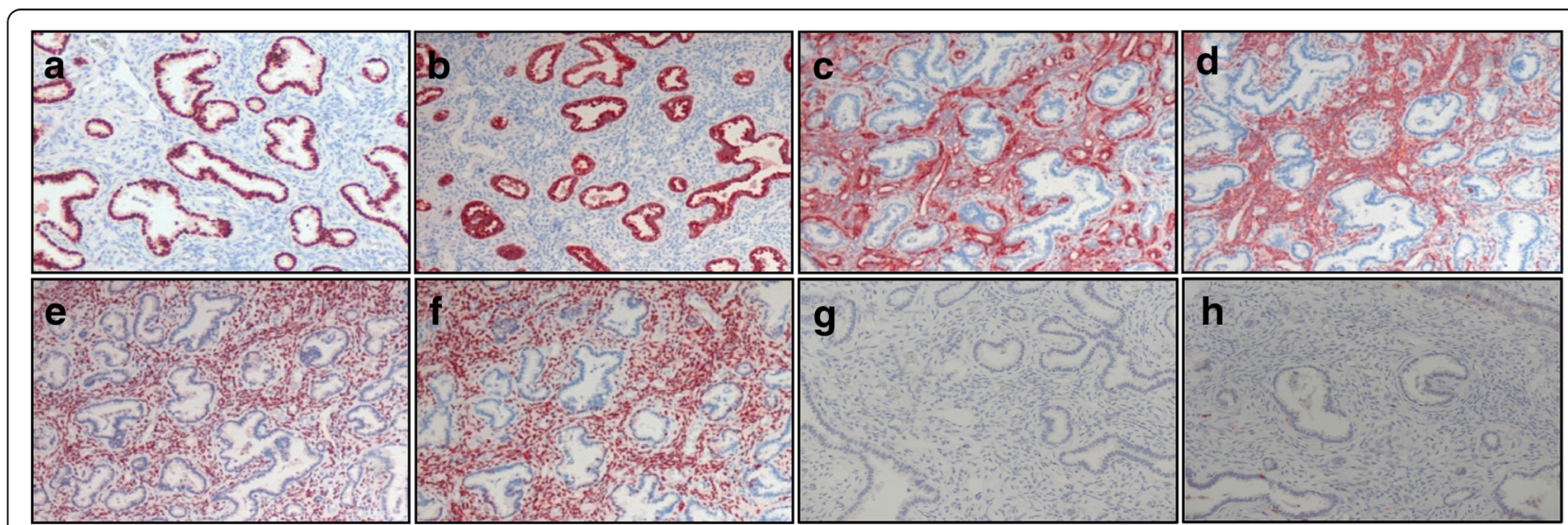

Fig. 3 Immuno-histochemical staining sections of the pulmonary nodule. Positive immunoreactivity for TTF-1 (a), EMA (b), SMA (c), desmin (d), ER (e), PR (f), HMB45 (g), and S100 (h) was observed. TTF-1: Thyroid transcription factor-1, EMA: Epithelial membrane antigen, SMA: Smooth muscle actin, ER: Estrogen receptor, PR: Progesterone receptor 
inactivation, and $12 \mathrm{q} 15$ rearrangements involving the HMGA2 [20, 21]. These gene analyses appear to be helpful for the correct diagnosis of BML and differential diagnosis.

To date, no standard treatment for BML has been introduced. Current therapeutic options include careful observation, surgical resection of pulmonary nodules, bilateral debulking oophorectomy, hormonal therapy, and radiation therapy to the ovary and chemotherapy [11]. In our patient, there was no mitotic figure in the tumor. Thus, we decided careful observation for pulmonary BML was critical.

\section{Conclusions}

In this report, we introduced an interesting case of pulmonary BML that manifested as multiple nodules with various radiologic features including cystic, mixed typed and solid natures. This radiologic presentation suggests that physicians should include pulmonary BML as a differential diagnosis when they encounter multiple pulmonary cystic nodules with diverse characteristics, especially in the case of middle-aged women with a history of uterine pathology.

\section{Abbreviations \\ BML: Benign metastasizing leiomyoma; CT: Computed tomography; EMA: Epithelial membrane antigen; ER: Estrogen receptor; LAM: Lymphangioleiomyomatosis; PLCH: Pulmonary Langerhans cell histiocytosis; PR: Progesterone receptor; SMA: Smooth muscle actin; TTF- 1: Thyroid transcription factor-1}

\section{Acknowledgments}

Not applicable.

\section{Funding}

This work was supported by the Korea Healthcare Technology R\&D Project, Ministry for Health and Welfare, Republic of Korea (Grant HI12C1786 and Grant HI16C0062), by the Basic Science Research Program through the National Research Foundation of Korea (NRF) funded by the Ministry of Science, ICT and future Planning (NRF-2014R1A2A1A01002823), and by the fund of Biomedical Research Institute, Chonbuk National University Hospital.

\section{Availability of data and materials}

Not applicable.

\section{Authors' contributions}

$Y C L$ and SRK designed the study. YHC, YL, and SYJ drafted the manuscript. YCL, SRK, SYJ, YHC and SYP contributed to the diagnosis and treatment. MJC reviewed and analyzed pathologic data. $Y C L$ and SRK reviewed and supervised the manuscript. All the authors approved the final version of the manuscript.

\section{Ethics approval and consent to participate}

The Institutional Review Board of Chonbuk National University Hospital stated that it was not necessary to achieve IRB approval for this case report, but that patient consent was required as the study dealt only with the retrospective use of the patient's medical record and related images.

\section{Consent for publication}

Written informed consent was obtained from the patient prior to the publication of this case report and accompanying images.

\section{Competing interests}

The authors declare that they have no competing interests.

\section{Publisher's Note}

Springer Nature remains neutral with regard to jurisdictional claims in published maps and institutional affiliations.

\section{Author details}

${ }^{1}$ Department of Internal Medicine, Chonbuk National University Medical School, San 2-20, Geumam-dong, Deokjin-gu, Jeonju, Jeonbuk 561-180, South Korea. ${ }^{2}$ Department of Pathology, Chonbuk National University Medical School, Jeonju, South Korea. ${ }^{3}$ Research Institute of Clinical Medicine of Chonbuk National University-Biomedical Research Institute of Chonbuk National University Hospital, Jeonju, South Korea.

Received: 23 September 2015 Accepted: 29 August 2017

Published online: 12 September 2017

\section{References}

1. Steiner PE. Metastasizing fibroleiomyoma of the uterus-a report of a case and review of the literature. Am J Pathol. 1939;15:89-110.

2. Pitts S, Oberstein EM, Glassberg MK. Benign metastasizing leiomyoma and lymphangioleiomyomatosis: sex-specific diseases? Clin Chest Med. 2004;25: $343-60$.

3. Kayser K, Zink S, Schneider T, Dienemann H, André S, Kaltner H, Schüring MP, Zick Y, Gabius HJ. Benign metastasizing leiomyoma of the uterus: documentation of clinical, immunohistochemical and lectin-histochemical data of ten cases. Virchows Arch. 2000;437:284-92

4. Goyle KK, Moore DF Jr, Garrett C, Goyle V. Benign metastasizing leiomyomatosis: case report and review. Am J Clin Oncol. 2003;26:473-6.

5. Esteban JM, Allen WM, Schaerf RH. Benign metastasizing leiomyoma of the uterus: histologic and immunohistochemical characterization of primary and metastatic lesions. Arch Pathol Lab Med. 1999:123:960-2.

6. Maredia R, Snyder BJ, Harvey LA, Schwartz AM. Benign metastasizing leiomyoma in the lung. Radiographics. 1998;18:779-82.

7. Martin E. Leiomyomatous lung lesions: a proposed classification. AJR Am J Roentgenol. 1983;141:269-72.

8. Shin MS, Fulmer JD, Ho KJ. Unusual computed tomographic manifestations of benign metastasizing leiomyomas as cavitary nodular lesions or interstitial lung disease. Clin Imaging. 1996;20:45-9.

9. Camenzuli A, Thwaite E, Huda B, Haqqani M, Warburton CJ, Curtis J. Cavitation in lung masses from benign metastizing leiomyomatosis. Clin Radiol Extra. 2004;59:83-5.

10. Moon H, Park SJ, Lee HB, Kim SR, Choe YH, Chung MJ, Jin GY, Lee YC Pulmonary Benign Metastasizing Leiomyoma in a Postmenopausal Woman. Am J Med Sci. 2009:338:72-4.

11. Rivera JA, Christopoulos S, Small D, Trifiro M. Hormonal manipulation of benign metastasizing leiomyomas: report of two cases and review of the literature. J Clin Endocrinol Metab. 2004;89:3183-8.

12. Nasu K, Tsuno A, Takai N, Narahara H. A case of benign metastasizing leiomyoma treated by surgical castration followed by an aromatase inhibitor, anastrozole. Arch Gynecol Obstet. 2009;279:255-7.

13. Rakhshani N, Hormazdi M, Abolhasani M, Shahzadi M. Benign metastasizing leiomyoma of the uterus. Arch Iran Med. 2007;10:97-9.

14. Osadchy A, Zehavi T, Zissin R. Pulmonary benign metastasising leiomyomas presenting as fluid-containing masses on CT in a patient with two unrelated malignancies. Br J Radiol. 2005:78:639-41.

15. Ryu JH, Swensen SJ. Cystic and Cavitary Lung Diseases: Focal and Diffuse. Mayo Clin Proc. 2003;78:744-52.

16. Prayson RA, Hart WR. Pathologic considerations of uterine smooth muscle tumors. Obstet Gynecol Clin N Am. 1995;22:637-57.

17. Kjerulff KH, Langenberg P, Seidman JD, Stolley PD, Guzinski GM. Uterine leiomyomas. Racial differences in severity, symptoms and age at diagnosis. J Reprod Med. 1996:41:483-90.

18. Matsui K, Takeda K, Yu ZX, Valencia J, Travis WD, Moss J, Ferrans VJ. Downregulation of estrogen and progesterone receptors in the abnormal smooth muscle cells in pulmonary lymphangioleiomyomatosis following therapy: an immunohistochemical study. Am J Respir Crit Care Med. 2000; 161:1002-9. 
19. Hoon V, Thung SN, Kaneko M, Unger PD. HMB-45 reactivity in renal angiomyolipoma and lymphangioleiomyomatosis. Arch Pathol Lab Med. 1994;118:732-4.

20. Nucci MR, Drapkin R, Dal Cin P, Fletcher CD, Fletcher JA. Distinctive cytogenetic profile in benign metastasizing leiomyoma: pathogenetic implications. Am J Surg Pathol. 2007:31:737-43.

21. Hodge JC, Morton CC. Genetic heterogeneity among uterine leiomyomata: insights into malignant progression. Hum Mol Genet. 2007;16:R7-R13.

Submit your next manuscript to BioMed Central and we will help you at every step:

- We accept pre-submission inquiries

- Our selector tool helps you to find the most relevant journal

- We provide round the clock customer support

- Convenient online submission

- Thorough peer review

- Inclusion in PubMed and all major indexing services

- Maximum visibility for your research

Submit your manuscript at www.biomedcentral.com/submit 